\title{
Textura de Bioprótese Mitral, Função Ventricular Esquerda e Formação de Trombo. Análise por Ecocardiografia Transesofágica e Aplicação de Bioescore
}

\author{
Texture of Mitral Bioprosthesis, Ventricular Function and Formation of Thrombus. \\ Analysis through Transesophageal Echocardiography and Use of Bioscore
}

Henry Abensur, Max Grinberg, José A. F. Ramires

São Paulo, SP

\section{Objetivo}

Diante da hipótese da participação do estresse mecânico como causa de disfunção de bioprótese mitral, decidimos avaliar a relação da preservação da textura dos folhetos da bioprótese mitral com a função ventricular esquerda, adicionalmente à eventual formação de trombo em átrio esquerdo nos pacientes com disfunção ventricular esquerda desde o implante da bioprótese mitral.

\section{Métodos}

Estudados 40 pacientes com bioprótese mitral por ecocardiograma transesofágico multiplanar, que foram divididos em dois grupos: com disfunção ventricular esquerda ( $F E=0,40 \pm 0.09)$ desde o implante da bioprótese ( 20 pacientes: idade $47,75 \pm 11,10$ anos e tempo de cirurgia $5,3 \pm 2,6$ anos) e com função ventricular esquerda normal $(F E=0,73 \pm 0.06)$ desde 0 implante (20 pacientes: idade $49,75 \pm 13,59$ anos e tempo de cirurgia $5,7 \pm 3$ anos). A textura dos folhetos da bioprótese foi analisada através de um escore ecocardiográfico transesofágico (Bioescore FACIME): 1) fusão de folhetos (escore 1 a 3); 2) aposição de tecidos (escore 1 a 3); 3) cálcio em folhetos (escore 1 a 5); 4) integridade dos folhetos (escore 1 a 3); 5) mobilidade dos folhetos (escore 1 a 4) e 6) Espessura dos folhetos (escore 1 a 3). A presença de trombos em átrio esquerdo foi avaliada pela varredura multiplanar do átrio e apêndice atrial esquerdos no estudo transesofágico.

\section{Resultados}

Não houve diferença significativa na textura dos folhetos da bioprótese mitral entre os dois grupos, tanto para o escore total $(8,7 \pm 2,4$ vs $7,9 \pm 2,1, p=0,259$ ), quanto para cada item analisado. Maior incidência de trombos encontrada em átrio e apêndice atrial esquerdos nos pacientes com disfunção ventricular ( $65 \%$ vs $20 \%, p=0,004)$.

\section{Conclusão}

A disfunção ventricular esquerda não foi fator protetor da textura dos folhetos da bioprótese em posição mitral no período pós-operatório tardio. Os pacientes com disfunção ventricular esquerda apresentam um ambiente mais propício à formação de trombos em átrio esquerdo.

\section{Palavras-chave}

bioprótese mitral, função ventricular esquerda, ecocardiografia transesofágica, formação de trombos

Instituto do Coração do Hospital das Clínicas - FMUSP

Correspondência: Henry Abensur - Rua Gaivota, 222/21 - 04522-030 -

São Paulo, SP - E-mail: henryab@terra.com.br

\section{Objective}

Facing the hypothesis of participation of mechanical stress as a cause of mitral bioprosthesis dysfunction, we decided to assess the relation of preservation of the texture of the mitral bioprosthesis leaflets with left ventricular function, in addition to the casual formation of thrombus in left atrium in patients with left ventricular dysfunction from the implant of mitral bioprosthesis.

\section{Methods}

Forty 40 patients with mitral bioprosthesis through multiplane transesophageal echocardiogram were studied and divided in two groups: with left ventricular dysfunction ( $F E=0.40 \pm 0.09$ ) since the bioprosthesis implant ( 20 patients: age $47.75 \pm 11.10$ years old and surgery time $5.3 \pm 2.6$ years) and with normal left ventricular function ( $F E=0.73 \pm 0.06)$ since the implant (20 patients: age $49.75 \pm 13.59$ years old and surgery time $5.7 \pm 3$ years). The texture of bioprosthesis leaflets was analyzed through a transesophageal echocardiographic score (FACIMT Bioscore): 1) Fusion of leaflets (score 1 to 3); 2) Apposition of tissues (score 1 to 3); 3) Calcium in leaflets (score 1 to 5 ); 4 ) Integrity of leaflets (score 1 to 3 ); 5 ) Motility of leaflets (score 1 to 4 ) and 6 ) Thickness of leaflets (score 1 to 3 ). The presence of thrombi in left atrium was assessed through multiplane scanning of the left atrium and left atrial appendage in the transesophageal study.

\section{Results}

There was no significant difference in the texture of bioprosthesis mitral position between the groups, for the total score $(8.7 \pm 2.4$ vs. $7.9 \pm 2.1, p=0.259)$, and for each analyzed item. A greater incidence of thrombi in left atrium and left atrial appendage was detected in patients with ventricular dysfunction ( $65 \%$ vs. $20 \%, p=0.004)$.

\section{Conclusion}

The left ventricular dysfunction was not a protecting factor of the texture of bioprosthesis leaflets in mitral position in the tardive postsurgery period. The patients with left ventricular dysfunction showed a more favorable environment for the formation of thrombi in left atrium.

\section{Key words}

mitral bioprosthesis, left ventricular function, transesophageal echocardiography, formation of thrombi 
Admite-se que o índice cardíaco pré-implante de bioprótese valvar mitral maior do que $2,0 \mathrm{~L} / \mathrm{min} / \mathrm{m}^{2}$ seja fator de aceleração do processo de degeneração estrutural dos folhetos valvulares ${ }^{1}$; esta hipótese sugere a participação do estresse mecânico como causa de disfunção da bioprótese $e^{2-4}$.

Observações de nossa prática clínica sugerem uma possível relação entre a preservação da textura dos folhetos da bioprótese mitral e a disfunção ventricular esquerda. Assim, portadores de disfunção ventricular esquerda, submetidos a menor tensão de fechamento dos folhetos, desde o implante da bioprótese mitral, de certa forma apresentariam menor desenvolvimento do processo degenerativo. Da mesma forma estes portadores de bioprótese mitral e disfunção ventricular esquerda, submetidos a um ambiente de maior estase pela disfunção ventricular esquerda, poderiam estar mais predispostos à formação de trombos intracavitários.

A introdução da modalidade transesofágica permitiu superar dificuldades técnicas apresentadas ao exame transtorácico, principalmente graças à proximidade do esôfago com o átrio esquerdo; ela permite a utilização de transdutores de maior freqüência, em ausência de estruturas que se interponham ao coração, resultando em imagens cardíacas de melhor qualidade de sinal e maior grau de resolução ${ }^{5,6,7}$. 0 método traz melhor definição quanto à textura e mobilidade dos folhetos, presença de massas anômalas, ou descontinuidade da sutura do anel da prótese ${ }^{8-10}$. Contamos atualmente com a tecnologia multiplanar, que oferece transdutores com capacidade de realizar todas as modalidades de Doppler, sendo também multifreqüenciais que produzem um contínuo de imagens transversas e longitudinais pela rotação do arranjo dos cristais, tornando mais fácil visibilizar imagens intermediárias fora do eixo entre os planos primários, transverso e longitudinal. Há, desta forma, aumento na qualidade das informações tomográficas obtidas em comparação com as obtidas pelos transdutores biplanares ${ }^{11-14}$.

0 método transesofágico é também relevante na detecção de trombos intracardíacos. A imagem pela ecocardiografia transtorácica tem sensibilidade limitada para o trombo atrial, em grande parte devido ao trombo forma-se, em geral, no apêndice atrial esquerdo, que é bem visibilizado pela modalidade transesofágica ${ }^{15-17}$.

0 objetivo desta pesquisa foi verificar através da ecocardiografia transesofágica multiplanar, se pacientes com disfunção ventricular esquerda desde o implante da bioprótese mitral teriam maior grau de preservação da textura de seus folhetos e maior risco de formação de trombos no átrio esquerdo, quando comparados a um grupo controle.

\section{Métodos}

Foram estudados 40 portadores de bioprótese mitral há mais de dois anos, acompanhados no Ambulatório da Unidade Clínica de Cardiopatias Valvares do Instituto do Coração. Vinte pacientes consecutivos (média de idade de $47,7 \pm 11,1$ anos) foram selecionados por apresentarem disfunção do ventrículo esquerdo desde o implante, verificada pela fração de ejeção menor que 0,45 (Modo unidimensional - método cubo) nos ecocardiogramas de acompanhamento, e constituíram o grupo função ventricular anormal (FVA). Outros 20 pacientes (media de idade de $49,7 \pm 13,6$ anos), com função ventricular esquerda normal (fração de ejeção maior que 0,65 ) desde 0 implante, foram pareados e compuseram 0 grupo controle de função ventricular esquerda normal (FVN). Pacientes com fração de ejeção entre 0,45 e 0,65 foram excluídos do estudo. Os dados clínicos, incluindo classe funcional, ritmo cardíaco, uso de medicação e controles laboratoriais do nível de anticoagulação foram obtidos pela análise de prontuários (tab. I).

Foram adicionalmente anotados o ritmo cardíaco (eletrocardiograma de 12 derivações), a utilização de terapia anticoagulante (dados de anamnese e prontuário) e tempo de normatização internacional (INR - método turbidimétrico, automatizado).

Os pacientes foram submetidos a ecocardiograma utilizandose aparelho Power Vision 7000 da Toshiba. Da modalidade transtorácica, obtiveram-se as variáveis: dimensão diastólica final do ventrículo esquerdo (DDFVE), dimensão sistólica final do ventrículo esquerdo (DSFVE), FE pelo método do $\mathrm{Cubo}^{18}$ e dimensão do átrio esquerdo realizada no final da ejeção do ventrículo esquerdo, de acordo com as recomendações da Associação Americana de Ecocardiografia ${ }^{19}$, tendo sido obtidas no modo unidimensional e guiadas pelo modo bidimensional. Com ajuda do mapeamento de fluxos em cores, foi verificada a existência de regurgitação das valvas aórtica e tricúspide. Na presença de regurgitação, esta era quantificada de forma qualitativa pelo mapeamento de fluxo em cores. Com a utilização do Doppler contínuo nos pacientes que apresentavam insuficiência tricúspide, obtivemos a diferença de pressão entre o ventrículo direito e 0 átrio direito, que, acrescida da pressão estimada do átrio direito, reflete a pressão sistólica do ventrículo direito e, por conseguinte, a pressão sistólica da artéria pulmonar $(\mathrm{PAP})^{20}$. A área da prótese mitral foi calculada pela utilização do Doppler Contínuo e da metodologia do tempo de meia pressão ${ }^{21}$. 0 gradiente através da valva aórtica foi calculado pelo Doppler contínuo, aplicando-se a equação modificada de Bernoulli22.

Ao término do ecocardiograma transtorácico, o paciente, que já havia sido orientado para estar em jejum há $4 \mathrm{~h}$, assinava o termo de consentimento pós-informação para a realização do ecocardiograma transesofágico multiplanar. 0 exame foi realizado segundo método já consagrado e estabelecido na praticam clinica ${ }^{11,23,24}$. Todos os pacientes receberam anestesia tópica, com solução anestésica local a base de lidocaína a 10\%, aplicada na orofaringe, palato duro e mole.

\begin{tabular}{|lccc|}
\hline \multicolumn{4}{|c|}{ Tabela I - Características clínicas e ecocardiográficas } \\
dos grupos estudados \\
\hline \\
\cline { 2 - 3 } Variáveis & Grupo & $\mathrm{p}$ \\
\cline { 2 - 3 } & $\mathrm{FVA}$ & $\mathrm{FVN}$ & \\
\hline Idade (anos) & $47,7 \pm 11,1$ & $49,7 \pm 13,6$ & $0,742^{(1)}$ \\
Sexo: feminino & $16(80 \%)$ & $11(55 \%)$ & $0,091^{(2)}$ \\
Peso (kg) & $68,4 \pm 19,4$ & $62,2 \pm 12,5$ & $0,242^{(1)}$ \\
Altura (cm) & $164,0 \pm 7,7$ & $159,7 \pm 8,7$ & $0,106^{(1)}$ \\
Tempo pós-cirurgia (anos) & $5,3 \pm 2,6$ & $5,7 \pm 3,0$ & $0,655^{(1)}$ \\
Diâmetro Diastólico (mm) & $68,6 \pm 12,4$ & $47,3 \pm 4,9$ & $0,001^{(1)}$ \\
Diâmetro Sistólico (mm) & $57,9 \pm 12,5$ & $30,7 \pm 4,6$ & $0,001^{(1)}$ \\
Fração de ejeção (cubo) & $0,40 \pm 0,09$ & $0,73 \pm 0,06$ & $0,001^{(1)}$ \\
FA & $17(85 \%)$ & $13(65 \%)$ & $0,144^{(2)}$ \\
Ritmo Sinusal & $3(15 \%)$ & $7(35 \%)$ & \\
Átrio esquerdo (mm) & $58,2 \pm 13,4$ & $50,6 \pm 8,6$ & $0,040^{(1)}$ \\
Terapia anticoagulante ausente & $15(75 \%)$ & $18(90 \%)$ & \\
Terapia anticoagulante presente & $5(25 \%)$ & $2(10 \%)$ & $0,407^{(3)}$ \\
\hline
\end{tabular}

(1) Nível descritivo de probabilidade do teste $t$ de Student; (2) nível descritivo de probabilidade do teste Qui-quadrado; (3) nível descritivo de probabilidade do teste exato de Fisher; FVA - função ventricular anormal; FVN - função ventricular normal; FA - fibrilação atrial. 
Após a introdução do transdutor no esôfago e a localização da bioprótese mitral, realizava-se a varredura da prótese em múltiplos planos (0 a 180).

Trombos e presença de formação de contraste espontâneo nos átrios e no apêndice atrial esquerdo foram observados através de varredura multiplanar.

Estimulados pela notória aplicabilidade do escore ecocardiográfico para decisões sobre valvuloplastia mitral por cateter balão ${ }^{25}$, desenvolvemos uma avaliação ecocardiográfica transesofágica das bioprótese em posição mitral, composta por seis itens; a qual denominamos FACIME, (acrônimo de fusão de folhetos, aposição de tecido, cálcio em folhetos, integridade dos folhetos, mobilidade dos folhetos e espessura dos folhetos - quadro 1 ).

A presença de insuficiência central da bioprótese ou periprotética foi analisada através do mapeamento de fluxo em cores.

Trombos e presença de formação de contraste espontâneo nos átrios e no apêndice atrial esquerdo foram observados através de varredura multiplanar.

Os estudos ecocardiográficos transtorácico e transesofágico foram gravados em fitas de VHS. Na leitura da fita era aplicado o Bioescore FACIME e analisados os itens do estudo ecocardiográfico delineados acima. A leitura da fita foi feita por dois observadores isoladamente e os dados discordantes foram resolvidos por consenso em uma terceira leitura realizada pelos dois observadores em conjunto.

Inicialmente todas as variáveis foram analisadas descritivamente. Para as variáveis contínuas, esta analise foi feita através da observação dos valores mínimos e máximos, e do cálculo de médias e desvio-padrão e medianas. Para as variáveis classificatórias, calcularam-se freqüências absolutas.

Para a analise da hipótese de igualdade de proporções entre os dois grupos, utilizou-se o teste Qui-quadrado, ou o teste exato de Fisher ${ }^{26}$. A hipótese de igualdade entre duas médias foi verificada fazendo uso do teste $t$ de student ${ }^{26}$. Para a variável escore total foi utilizado 0 teste não-paramétrico de Mann-Whitney ${ }^{26}$. Para a comparação do escore total entre vários grupos foi utilizado 0 teste não-paramétrico de Kruskal-Wallis ${ }^{26}$.

O nível de significância utilizado para os testes foi de $5 \%$.

\section{Resultados}

Os dois grupos foram semelhantes em relação à área da bioprótese mitral, presença de insuficiência central e periprotética, à presença de estenose aórtica, à presença de insuficiência tricúspide e ao valor estimado da pressão sistólica da artéria pulmonar (tab. II). No grupo FVA foram detectados dois casos de insuficiência periprotética, visibilizado somente no estudo transesofágico.
A aplicação do Bioescore FACIME não identificou diferença significativa quanto ao escore total $(8,70 \pm 2,39$ e 7,95 $\pm 2,14$, $p=0,259)$ entre os grupos FVA e FVN. Análise individualizada dos itens do Bioescore FACIME também não mostrou diferenças significativas (tab. III).

Trombos em átrio esquerdo foram encontrados no grupo FVA em proporção maior do que no FVN. O grupo FVA apresentou trombos em 10, 20 e 35\%, respectivamente, em apêndice atrial esquerdo, átrio esquerdo e apêndice atrial esquerdo e átrio esquerdo simultâneos. No grupo FVN trombos foram encontrados na distribuição de 10, 5 e $5 \%$ (tab. IV).

Contraste espontâneo esteve presente em $85 \%$ dos pacientes do grupo FVA e em $60 \%$ do grupo FVN, mostrando uma tendência estatística mais favorável à formação de contraste espontâneo no grupo FVA (tab. IV).

0 emprego da terapia anticoagulante foi semelhante nos dois grupos. No grupo FVA, $75 \%$ dos pacientes não estavam recebendo anticoagulantes orais, e, no grupo $\mathrm{FVN}, 90 \%$ também não estavam, apenas três tinham índice de normatização internacional (INR) atualizados na época do estudo, sendo que nenhum deles estava adequadamente anticoagulados. No grupo FVN, dois pacientes apenas recebiam terapia anticoagulante oral, estando com INR de 3,4 e 4,7, respectivamente.

0 ritmo de fibrilação atrial esteve presente em 85 e $65 \%$ nos pacientes dos grupos FVA e FVN, respectivamente. Não houve diferenças estatísticas entre estes valores.

A dimensão do átrio esquerdo foi significantemente maior no grupo FVA em relação ao grupo FVN $(58,2 \pm 13,4$ versus $50,6 \pm$ $8,6 \mathrm{~mm}, \mathrm{p}=0,040$ ).

\section{Discussão}

A evolução das biopróteses está bem caracterizada por meio de curvas atuariais. As alterações estruturais de repercussão clínica das biopróteses ocorrem, em média, entre 5 a 10 anos após 0 seu implante, com estenose devido à calcificação e espessamento dos folhetos, e incompetência pela inadequada justaposição das comissuras nos sítios de infiltração de cálcio ${ }^{27-30}$. Pareceu-nos importante elaborar um escore, no qual, de maneira quantitativa, pudéssemos documentar este fenômeno biológico da degeneração das biopróteses, dando ênfase aos aspectos que mais são analisados na literatura em relação aos folhetos das biopróteses. Utilizamos, o acrônimo FACIME, seis distintas letras com as quais pretendemos facilitar a memorização dos itens do bioescore: $\mathrm{F}$ de fusão de folhetos, $\mathrm{A}$ de aposição de tecido, $\mathrm{C}$ de cálcio de folhetos, I de integridade dos folhetos, $\mathrm{M}$ de mobilidade dos folhetos e

\begin{tabular}{|c|c|c|c|c|c|}
\hline \multicolumn{6}{|c|}{ Quadro 1 - Bioescore FACIME } \\
\hline Graus variáveis & 1 & 2 & 3 & 4 & 5 \\
\hline Fusão de folhetos & Normal & Fusão de 2 folhetos & Fusão dos 3 folhetos & & \\
\hline Aposição de tecido & Normal & Por imagem filiforme & $\begin{array}{c}\text { Por trombo, } \\
\text { vegetação ou pannos }\end{array}$ & & \\
\hline Cálcio em folhetos & Normal & 1 - 2 pontos de cálcio & $>2$ pontos de cálcio & Segmentar em folheto & Segmentar em mais de 2 folhetos \\
\hline Integridade dos folhetos & Normal & $\begin{array}{l}\text { Perfuração de } 1 \text { ou } \\
\text { mais folhetos }\end{array}$ & $\begin{array}{l}\text { Ruptura de } 1 \text { ou } \\
\text { mais folhetos }\end{array}$ & & \\
\hline Mobilidade dos folhetos & Normal & Diminuída em 1 folheto & Diminuída em 2 folhetos & Diminuída em 3 folhetos & \\
\hline Espessura dos folhetos & Normal & $2-4 \mathrm{~mm}$ & $>4 \mathrm{~mm}$ & & \\
\hline
\end{tabular}




\begin{tabular}{|c|c|c|c|}
\hline \multicolumn{4}{|c|}{ Tabela II - Características ecocardiográficas da série } \\
\hline \multirow[t]{2}{*}{ Variáveis } & \multicolumn{2}{|c|}{ Grupo } & \multirow[t]{2}{*}{$\mathrm{p}$} \\
\hline & FVA & FVN & \\
\hline Área da prótese $-\mathrm{cm}^{2}$ & $2,6 \pm 0,7$ & $2,4 \pm 0,4$ & $0,378^{(1)}$ \\
\hline \multicolumn{4}{|l|}{ Insuficiência central } \\
\hline Ausente & $13(65 \%)$ & $14(70 \%)$ & $0,736^{(2)}$ \\
\hline Discreta & $7(35 \%)$ & $6(30 \%)$ & \\
\hline \multicolumn{4}{|l|}{ Insuficiência periprotética } \\
\hline Ausente & $18(90 \%)$ & $20(100 \%)$ & $0,487^{(3)}$ \\
\hline Discreta & $1(5 \%)$ & $0(0 \%)$ & \\
\hline Moderada & $1(5 \%)$ & $0(0 \%)$ & \\
\hline \multicolumn{4}{|l|}{ Insuficiência aórtica } \\
\hline Ausente & $9(45 \%)$ & $7(35 \%)$ & $0,300^{(3)}$ \\
\hline Discreta & $9(45 \%)$ & $13(65 \%)$ & \\
\hline Moderada & $2(10 \%)$ & $0(0 \%)$ & \\
\hline \multicolumn{4}{|l|}{ Estenose aórtica } \\
\hline Ausente & $19(95 \%)$ & $18(90 \%)$ & $1,000^{(3)}$ \\
\hline Discreta & $1(5 \%)$ & $2(10 \%)$ & \\
\hline \multicolumn{4}{|l|}{ Insuficiência tricúspide } \\
\hline Ausente & $3(15 \%)$ & $3(15 \%)$ & $1,000^{(3)}$ \\
\hline Discreta & $10(50 \%)$ & $10(50 \%)$ & \\
\hline Moderada & $5(25 \%)$ & $6(30 \%)$ & \\
\hline Importante & $2(10 \%)$ & $1(5 \%)$ & \\
\hline $\begin{array}{l}\text { Pressão sistólica da } \\
\text { artéria pulmonar - mmHg }\end{array}$ & $42,4 \pm 10,7$ & $39,8 \pm 10,1$ & $0,485^{(1)}$ \\
\hline \multicolumn{4}{|c|}{$\begin{array}{l}\text { (1) Nível descritivo de probabilidade do teste t de Student; (2) nível } \\
\text { descritivo de probabilidade do teste Qui-quadrado; (3) nível descritivo de } \\
\text { probabilidade do teste exato de Fisher; FVA - função ventricular anormal; } \\
\text { FVN - função ventricular normal. }\end{array}$} \\
\hline
\end{tabular}

\begin{tabular}{|c|c|c|c|c|}
\hline \multicolumn{5}{|c|}{ Tabela III - Bioescore FACIME - Resultados } \\
\hline Itens do bioescore & Escore & Grupo FVA & Grupo FVN & $\mathrm{p}$ \\
\hline \multirow[t]{3}{*}{ Fusão de folhetos } & 1 & $18(90 \%)$ & $20(100 \%)$ & $0,487^{(3)}$ \\
\hline & 2 & $2(10 \%)$ & $0(0 \%)$ & \\
\hline & 3 & $0(0 \%)$ & $0(0 \%)$ & \\
\hline \multirow[t]{3}{*}{ Aposição de tecidos } & 1 & $17(85 \%)$ & $18(90 \%)$ & $0,605^{(3)}$ \\
\hline & 2 & $2(10 \%)$ & $0(0 \%)$ & \\
\hline & 3 & $1(5 \%)$ & $2(10 \%)$ & \\
\hline \multirow[t]{5}{*}{ Cálcio em folhetos } & 1 & $6(30 \%)$ & $5(25 \%)$ & $0,837^{(3)}$ \\
\hline & 2 & $11(55 \%)$ & $13(65 \%)$ & \\
\hline & 3 & $1(5 \%)$ & $0(0 \%)$ & \\
\hline & 4 & $1(5 \%)$ & $0(0 \%)$ & \\
\hline & 5 & $1(5 \%)$ & $2(10 \%)$ & \\
\hline \multirow[t]{3}{*}{ Integridade dos folhetos } & os 1 & $20(100 \%)$ & $20(100 \%)$ & - \\
\hline & 2 & $0(0 \%)$ & $0(0 \%)$ & \\
\hline & 3 & $0(0 \%)$ & $0(0 \%)$ & \\
\hline \multirow[t]{4}{*}{ Mobilidade dos folhetos } & os 1 & $11(55 \%)$ & $17(85 \%)$ & $0,166^{(3)}$ \\
\hline & 2 & $5(25 \%)$ & $2(10 \%)$ & \\
\hline & 3 & $4(20 \%)$ & $1(5 \%)$ & \\
\hline & 4 & $0(0 \%)$ & $0(0 \%)$ & \\
\hline \multirow[t]{3}{*}{ Espessura dos folhetos } & 1 & $9(45 \%)$ & $12(60 \%)$ & $0,693^{(3)}$ \\
\hline & 2 & $7(35 \%)$ & $4(20 \%)$ & \\
\hline & 3 & $2(25 \%)$ & $3(15 \%)$ & \\
\hline Bioescore total & & $8,7 \pm 2,4$ & $7,9 \pm 2,1$ & $0,259^{(4)}$ \\
\hline \multicolumn{5}{|c|}{$\begin{array}{l}\text { (3) Nível descritivo de probabilidade do teste exato de Fisher; (4) nível } \\
\text { descritivo de robabilidade do teste de Mann-Whiney; FVA - função } \\
\text { ventricular anormal; FVN - função ventricular normal. }\end{array}$} \\
\hline
\end{tabular}

E de espessura dos folhetos. Esperamos, desta forma, nos valer de um método de diagnóstico apropriado que seja uma linguagem de comunicação homogênea entre clínicos e cirurgiões.

0 tempo médio de implante das biopróteses foi de 5,3 $\pm 2,6$ e $5,7 \pm 3$ anos, respectivamente, para os grupos FVA e FVN. Com mais de cinco anos de implante das biopróteses havia 10 e 8 pacientes, respectivamente, para os grupos FVA e FVN.

A disfunção ventricular esquerda não foi fator de proteção da textura dos folhetos da bioprótese mitral, pois, tanto o valor do

\begin{tabular}{|c|c|c|c|}
\hline \multicolumn{4}{|c|}{$\begin{array}{c}\text { Tabela IV - Aspectos ecocardiográficos relacionados à } \\
\text { trombose atrial esquerda }\end{array}$} \\
\hline \multirow[t]{2}{*}{ Variável } & \multicolumn{2}{|c|}{ Grupo } & \multirow[t]{2}{*}{$\mathrm{p}$} \\
\hline & FVA & FVN & \\
\hline Trombo Ausente & $7(35 \%)$ & $16(80 \%)$ & $0,012^{(3)}$ \\
\hline \multicolumn{4}{|l|}{ Trombo Presente } \\
\hline AAE & $2(10 \%)$ & $2(10 \%)$ & \\
\hline $\mathrm{AE}$ & $4(20 \%)$ & $1(5 \%)$ & \\
\hline$A E-A A E$ & $7(35 \%)$ & $1(5 \%)$ & \\
\hline Total & $13(65 \%)$ & $4(20 \%)$ & $0,004^{(2)^{*}}$ \\
\hline \multicolumn{4}{|c|}{ Contraste espontâneo } \\
\hline Ausente & $3(15 \%)$ & $8(40 \%)$ & \\
\hline Presente & $17(85 \%)$ & $12(60 \%)$ & $0,077^{(2)}$ \\
\hline \multicolumn{4}{|c|}{$\begin{array}{l}\text { Nível descritivo de probabilidade do teste t de Student; ( } 2 \text { ) nível descri- } \\
\text { tivo de probabilidade do tese Qui-quadrado; (3) nível descritivo de } \\
\text { probabilidade do teste exato de Fisher; }{ }^{*} \text { ausente versus presente; FVA } \\
\text { - função ventricular anormal; FVN - função ventricular normal; AAE - } \\
\text { apêndice atrial esquerdo; AE - átrio esquerdo. }\end{array}$} \\
\hline
\end{tabular}

escore total, como o de cada item do Bioescore FACIME, mostraram-se semelhantes nos dois grupos. Na literatura estudada, existe uma única referência sobre função ventricular esquerda e textura dos folhetos ${ }^{1}$, onde foi observada aceleração do processo de disfunção das biopróteses nos pacientes com índice cardíaco maior do que 2,0 L/min/ $/ \mathrm{m}^{2}$, na tentativa de explicar a menor durabilidade das biopróteses em pacientes mais jovens.

A análise individual dos itens do Bioescore FACIME torna-se útil: por exemplo, o paciente $\mathrm{n} 023$ do grupo FVN e 5 do grupo FVA obtiveram escore total igual a 12. Contudo, o paciente 5 do grupo FVA obteve 3 no item aposição de tecido e o paciente 23 do grupo FVN obteve, neste item, 1 . Desta maneira o paciente 5 do grupo FVA tinha trombose de um dos seus folhetos, situação esta que determina conduta terapêutica específica. Portanto, é essencial a análise individual de cada item do escore para conhecermos a real situação da textura dos folhetos da bioprótese. Esta situação foi presenciada quando da utilização do escore ecocardiográfico da valva mitral para valvoplastia mitral por cateter balão, em que foi demonstrado que o item aparelho subvalvar era de importância prognostica maior para o sucesso do procedimento; não obstante, esta hierarquia não invalida a utilização do escore rotineiramente ${ }^{31}$.

Só foi possível identificar as alterações da textura dos folhetos, com a utilização do método transesofágico. 0 estudo pela ecocardiografia transtorácica tem seu valor, principalmente, na análise dos fatores funcionais relacionados às biopróteses, como área, gradientes, presença de regurgitações e alterações grossei-

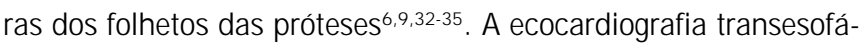
gica, pela utilização de transdutores de maior freqüência e pela maior proximidade das estruturas analisadas, nos permite uma avaliação fina da textura dos folhetos, uma quantificação mais precisa do grau das regurgitações das biopróteses mitrais e a detecção de complicações, como vegetações, trombos e regurgitações periprotéticas ${ }^{5,6,10,36,37}$. Nesta casuística, foi detectada insuficiência periprotética em dois pacientes do grupo FVA, não detectadas ao ecocardiograma transtorácico, situação esta demonstrada por Khanderia e cols. ${ }^{36}$.

A posição mitral da bioprótese favorece a degeneração primaria dos folhetos mais precocemente, provavelmente por um maior estresse de fechamento, ocorrida na posição mitral durante a sísto$\mid \mathrm{e}^{27,38-40}$. Na posição mitral, o tempo de abertura dos folhetos chega 
a ser três vezes o tempo de abertura dos folhetos da bioprótese aórtica ${ }^{41}$. Quando o ritmo é sinusal existe duplo movimento de abertura dos folhetos da bioprótese mitral; em um estudo realizado não foi demonstrada relação entre o tipo de ritmo e a degeneração primária da bioprótese ${ }^{42}$. Até o presente momento não existe confirmação em relação ao tamanho da bioprótese e a prevalência de degeneração primária da prótese. Quanto ao sexo, também não existe consenso. Neste estudo foi demonstrado que o fator função ventricular esquerda não exerceu influência na textura da bioprótese.

Trombo em átrio esquerdo e em seu apêndice foi dominante no grupo FVA ( $65 \%$ contra $20 \%, p=0,004)$, porém não houve diferença significativa em relação ao tipo de ritmo (fibrilação atrial ou sinusal) e quanto à terapia anticoagulante nos grupos estudados. Ficou demonstrado que os pacientes com disfunção ventricular esquerda apresentam um ambiente mais propício à formação de trombos em átrio esquerdo e apêndice atrial esquerdo. Em relação ao tamanho do átrio esquerdo, o grupo FVA apresentou o átrio esquerdo um pouco maior $(58,2 \pm 13,4$ vs $50,6 \pm 8,6 \mathrm{~mm}, \mathrm{p}=0,040)$.

Edmunds e cols. ${ }^{43}$ mostraram que houve necessidade de anticoagulação em 40 a $60 \%$ dos pacientes com prótese biológica em posição mitral; que a incidência de episódios tromboembólicos é maior nos primeiros três meses de implante das biopróteses; que a fibrilação atrial aumenta o risco de complicações tromboembólicas; que ainda não está claro o papel da presença de trombos atriais, do tamanho do átrio esquerdo e da história de eventos embólicos prévios, no aumento da incidência de eventos tromboembólicos. A fibrilação atrial é o principal fator identificado na literatura responsável pelo aumento do risco de tromboem- bolismo sistêmico nos pacientes com valvopatia mitral ${ }^{44-47}$. Relatos na literatura mostram menor incidência de eventos tromboembólicos nos pacientes com biopróteses de pericárdio bovino ou dura-máter em comparação com as biopróteses porcinas ${ }^{48-50}$.

Tamanho do átrio esquerdo, idade maior que 60 anos, possivelmente a disfunção ventricular esquerda e a hipertensão arterial sem tratamento somam riscos para tromboembolismo em pacientes com fibrilação atrial ${ }^{51}$. Estes pacientes devem receber terapia anticoagulante prolongada, mantendo um índice de normatização internacional entre 2,0 e 3,0. Por motivos provavelmente sociais, a maioria de nossos pacientes não estava com a anticoagulação adequadamente controlada.

Contraste espontâneo em átrio esquerdo e apêndice atrial esquerdo foi mais freqüente no grupo FVA, porém este dado não alcançou significância estatística ( $85 \%$ contra $60 \%, p=0,077$ ). Destaque-se que a presença de contraste espontâneo, fibrilação atrial e aneurisma do septo interatrial são fatores positivos independentes preditores da presença de trombos em átrio esquerdo e eventos cérebro-vasculares; em adição, a dilatação do átrio esquerdo e eventos cérebro-vasculares são preditores positivos independentes da presença de contraste espontâneo e de trombos em átrio esquerdo $0^{52}$.

Concluimos que a função ventricular esquerda não foi fator determinante da evolução da textura dos folhetos de bioprótese mitral, o Bioescore FACIME mostrou-se útil na análise evolutiva da textura dos folhetos da bioprótese mitral e a disfunção ventricular esquerda associou bioprótese mitral à maior incidência de formação de trombos em átrio esquerdo e apêndice atrial esquerdo.

\section{Referências}

1. Magilligan DJ , Lewis J W, Stein P, Alam M. The porcine bioprosthetic heart valva: experience at 15 years. Ann Thorac Surg 1989; 48:324-30.

2. Sabbah HN, Hamid MS, Stein PD. Mechanical stress on closed cusps of porcine bioprosthetic valves: correlation with sites of calcification. Ann Thorac Surg 1986; 42:93-6.

3. Thubrikar MJ , Deck J D, Aovad J, Nolan SP. Role of mechanical stress in calcification of aortic bioprosthetic valves. J Thorac Cardiovasc Surg 1983; 86:115-25.

4. Stein PD, Sabbah HN, Magilligan DJ J r. Can we delay the ocurrence of spontaneous degeneration of bioprosthetic valves? J Thorac Cardiovasc Surg 1988;96:343.

5. Nelessen U, Schnittger I, Appleton CP. Transesophageal two-dimensional echocardigraphy and color Doppler flow velocity mapping in the evaluation of cardiac valve prastheses. Circulation 1988; 78:848-55.

6. Chaudhry FA, Herrera C, Defrino PF, Mehlman DJ , Zabalgoitia M. Pathologic and angiographic correlations of transesophageal echocardigraphy in prosthetic heart valve dysfunction. Am Heart] 1991;122:1057-64.

7. Herrera CJ, Chaudhry FA, Defrino PF. Value limitations of transesophageal echocardiography in evaluating prosthetic or bioprosthetic valve dysfunction. AmJ Cardiol 1992; 69:697-9.

8. Khandheria BK. Transesophageal echocardiography in the evaluation of prosthetic valves. Cardiol Clin 1993; 11:427-36.

9. Daniel WG, Mügge A, Grote J. Comparison of transthoracic and transesophageal echocardiography for detection of abnormalities of prosthetic and bioprosthetic valves in the mitral and aortic positions. Am J Cardiol 1993; 71:210-15.

10. Groundstroem RD, Hoffman P, Bloomfield P, Sutherland GR. Additional value of biblane transesophageal imaging in assesment of mitral valve prostheses. BrHeart J 1993; 70:259-65.

11. Freeman WK, Seward JB, Khanderia BK. Transesophageal echocardiography Boston, Little Brown, 1993.

12. Pandian NG, Hsu TL, Schwartz SL et al. Multiplane transesophageal echocardiography. Imaging planes, echocardigraphic anatomy, and clinical experience with a prototype phased array OmniPlane probe. Echocardiography. Echocardiography 1992; 9:649-66.

13. Roelandt JR, Thomsom IR, VletterWB. Multiplane transesophageal echocardiography: latest evolution in imaging revolution. J Am Soc Echocardiogr 1992; 5: 361-7. diography: image orientation technique, anatomic correlations, and clinical applications. Mayo Clin Proc 1993; 68:1-29.

15. Aschenberg W, Schluter M, Kremer P. Transesophageal two-dimensional echocardiography for the detection of left atrial appendage thrombus. J Am Coll Cardiol 1986; 7: 163-7.

16. Dressler FA, Labovitz AJ . Systemic arterial emboli and cardiac masses: assesment with transesophageal echocardiography. Cardiol Clin 1993; 11: 447-60.

17. Olsen J D, Goldenberg IF, Pederson W. Exclusion of atrial thrombus by transesophageal echocardiography. J Am Soc Echocardiogr 1992; 5: 52-6.

18. Triulzi MO, Wilkins GT, Gillam LD. Normal adult cross-sectional echocardiographic valves: LV volumes. Echocardiography 1985; 2:153-70.

19. Sahn DJ , Demaria A, Kisslo J, Weyman A. The commitee on M-mode standartization of the American Society of Echocardiography. Recommendations regarding quantitation in M-mode echocardiographic measurements. Circulation 1978; 58: 1072.

20. Yock PG, Popp RL. Non-invasive estimation of right ventricular systolic pressure by Doppler ultrasound in patients with tricuspid regurgitation. Circulation 1984; 70: 657-62

21. Hatle L, Angelsen B, Tromsdal B. Nom-invasive assesment of pressure half-time by Doppler ultrasound. Circulation 1980; 60:1096.

22. Hatle L, Angelsen B, Tromsdal B. Noninvasive assesment of aortic stenosis by Doppler ultrasound. BrHeart] 1980; 43: 284.

23. Seward J B, Khandheria BK, Edwards WD. Biplanar transesophageal echocardiography: anatomic correlations, image orientation, and clinical applications. Mayo Clin. Proc., v.65, p.1193-213, 1990

24. Seward J B, Khandheria BK, Freeman WK. Multiplane transesophageal echocardiography: image orientation, examination technique, anatomic correlations, and clinical applications. Mayo Clin Proc 1993, 68:1-29.

25. Wilkins GT, Weyman AE, Abascal VM. Percutaneous balloon dilatation of mitral valve. Analysis of echocardiographic variables related to outcome and the mechanism of dilatation. Br Heart J 1988; 60: 299-308.

26. Rosner B. Fundamentals of biostatistics. $2^{\text {nd }}$ ed. Boston, PWS Publishers, Second edition, 1986.

27. J ones EL, Weintraub WS, Craver J M et al. Tem-year experience with the porcine bioprosthetic valve: interrelationship of valve survival and patient survival in 1,050 valve replacements. Ann Thorac Surg 1990; 49: 370-84. 
28. Pelletier LC, Carrier M, Leclerc Y, Lepage G, Deguise P, Dyrda I. Porcine versus pericardial bioprostheses: a comparison of late results in 1,583 patients. Ann Thorac Surg 1989; 47:352-61.

29. Bortolotti U, Milano A, Thiene G. Early mechanical failures of the Hancock pericardial xenograft. J Thorac Cardiovasc Surg 1987; 94: 200-07.

30. Cohn LH, Allred EN, Disesa V]. Early and late risk of aortic valve replacement: a 12 year concomitant comparison of the porcine bioprosthetic and tilting disc prosthetic aortic valves. J Thorac Cardiovasc Surg 1984; 88:695-705.

31. Medeiros CCJ , Moraes AV, Cardoso LF. São os componentes do aparelho valvar mitral de mesmo valor preditivo na valvoplastia mitral por cateter-balão? Estudo ecocardiográfico. Arq Bras Cardiol 1991; 57:11-20.

32. Cooper DM, Stewart WJ, Schiavone WA et al. Evalation of normal prosthetic valve function by Doppler echocardiography. Am Heart] 1987; 114: 576 -82.

33. Alam M, Lakier J B, Pickard SD, Goldstein S. Echocardiographic evaluation of porcine bioprosthetic valves: experience with 309 normal and 59 dysfunctioning valves. Am Heart] 1983; 52:309-15.

34. Formann MB, Phelan BK, Robertson RM, Virmani R. Correlation of two-dimensional echocardiography and pathologic findings in porcine valve dysfunction. J Am Coll Cardiol 1985; 5: 224-30.

35. Almeida J, Sepúlveda F, Gomes MR. Valor diagnóstico da ecocardiografia transesofágica no estudo das disfunções das próteses mitrais. Rev Port Cardiol 1993; 12: 155-61.

36. Khandheria BK, Seward J B, Oh J K. Value and limitations of transesophageal echocardiography in assesment of mitral valve prostheses. Circulation 1991; 83: 1956-68.

37. Scott PJ, Ettles DF, Wharton GA, Williams GJ . The value of transesophageal echocardiography in the investigation of acute prosthetic valve dysfunction. Clin Cardiol 1990; 13: 541-4.

38. Jamieson WRE, Hayden RI, Miyagishima RT et al. The Carpentier-Edwards standard porcine bioprosthesis: clinical performance to 15 years. J Cardiac Surg 1991; 6: S550-6.

39. O'brien MF, Stafford EG, Gardner MAH, Pohlner PG, Tesar PJ , Kear L, Smith SE. The Medtronic intact xenograft: analysis of 342 patients over a seven-year follow-up period. Ann Thorac Surg 1995; 60: S253-7.
40. Akins CW, Carrol DL, Buckley MJ, Daggett WM, Hilgenberg AD, Austen WG. Late results with Carpentier- Edwards porcine bioprosthesis. Circulation 1990; 82 (supp 4):IV-65-IV-74

41. Thurbrikar MJ, Deck DJ, Aouad J. Role of mechanical stress in calcification of aortic bioprosthetic valve. J Thorac Cardiovasc Surg 1983; 86:115.

42. Pansini S, Ottino G, Caimmi F, Del Ponte S, Morea M. Risk factors of primary tissue failure within the $11^{\text {th }}$ postoperative year in 217 patients with porcine bioprostheses. J Card Surg 1991; 6: S644-8.

43. Edmunds LH. Thromboembolic complications of current cardiac valvular prostheses. Ann Thorac Surg 1982; 34: 96-106.

44. Garcia-Bengechea J B, González-J uanatey J R, Rubio J, Durán D, Sierra J . Thromboembolism in patients with pericardial valves in the absence of chronic anticoagulation: 12 years' experience. Eur J Cardiothorac Surg 1991; 5: 592-7.

45. Askey J M, Bernstein S. The management of rheumatic heart disease in relation to systemic arterial embolism. Prog Cardiovasc Dis 1960; 3: 220-32.

46. Bannister $R$. The risks of deferring valvotomy in patients with moderate mitral stenosis. Lancet 1960; 2: 329-32.

47. Coulshed N, Epstein EJ , Mckendrick CS, Galloway RW, Walker E. Systemic embolism in mitral valve disease. Br HeartJ 1970; 32: 26-34.

48. Silverton NP, Tandon AD, Ionescu MI. Trombosis embolism and anticoagulant-related hemorrhage in mitral valve disease and mitral valve replacement. In: Ionescu MI, Cohn LH. Mitral valve disease diagnosis and treatment. London, Butteworths, 1984, p.337-45.

49. Zerbini EJ, Puig LB. The dura mater allograft valve. In: Ionescu MI. Tissue heart valves. London, Butteworths, 1979. P.253-301.

50. Pomerantzeff EJ , Zerbini EJ , Verginelli G, J atene AD. Valve replacement in the Heart Institute, University of São Paulo, Brazil. Ann Thorac Surg 1989; 48: S41-44.

51. Heras M, Chesebro J H, Fuster V et al. High risk of thromboemboli early after bioprosthetic cardiac valve replacement. J Am Coll Cardiol 1995; 25: 1111-19.

52. Kamensky G, Drahos P, Plevová N. Left atrial spontaneous echo contrast: its prevalence and importance in patients undergoing transesophageal echocardiography and particularly those with a cerebrovascular embolic event. J Am Soc Echocardiogr 1996; 9: 62-70. 